\title{
Development of a quantitative SRM-based proteomics method to study iron metabolism of Synechocystis sp. PCC 6803
}

Linda Vuorijoki ${ }^{1}$, Janne Isojärvi ${ }^{1}$, Pauli Kallio ${ }^{1}$, Petri Kouvonen ${ }^{2}$, Eva-Mari Aro ${ }^{1}$, Garry Corthals $^{2,3}$, Patrik R. Jones ${ }^{4}$ and Dorota Muth-Pawlak ${ }^{1,2}$

${ }^{1}$ Molecular Plant Biology Unit, Department of Biochemistry, University of Turku, Finland, ${ }^{2}$ Turku Proteomics Facility, Centre for Biotechnology, University of Turku and Åbo Akademi University, Turku, Finland, ${ }^{3}$ Van't Hoff Institute for Molecular Sciences, University of Amsterdam, Amsterdam, The Netherlands, ${ }^{4}$ Department of Life Sciences, Imperial College London, Sir Alexander Fleming Building, London SW7 2AZ, United Kingdom

Corresponding author: Dorota Muth-Pawlak ${ }^{1,2}$

\section{Table of contents:}

Supporting data: Links to the results in public databases

Figure S-1: Growth of Synechocystis sp. PCC 6803 under iron deprivation.

Figure S-2. In vivo absorbance spectrum

Figure S-3 Standard curve representing retention times of the iRT-peptides in the initial alignment run.

Figure S-4 Abundance of 252 peptides with CVs calculated for 3 biological replicates of wild type in standard condition.

Figure S-5 Estimation of significance of the expression level change (FC) in the experiment. 
Supporting data: Links to the results in public databases

Panorama

https://panoramaweb.org/labkey/Vuorijoki et al 2015.url

PASSEL

Identifier: PASS00726

https://db.systemsbiology.net/sbeams/cgi/PeptideAtlas/PASS View

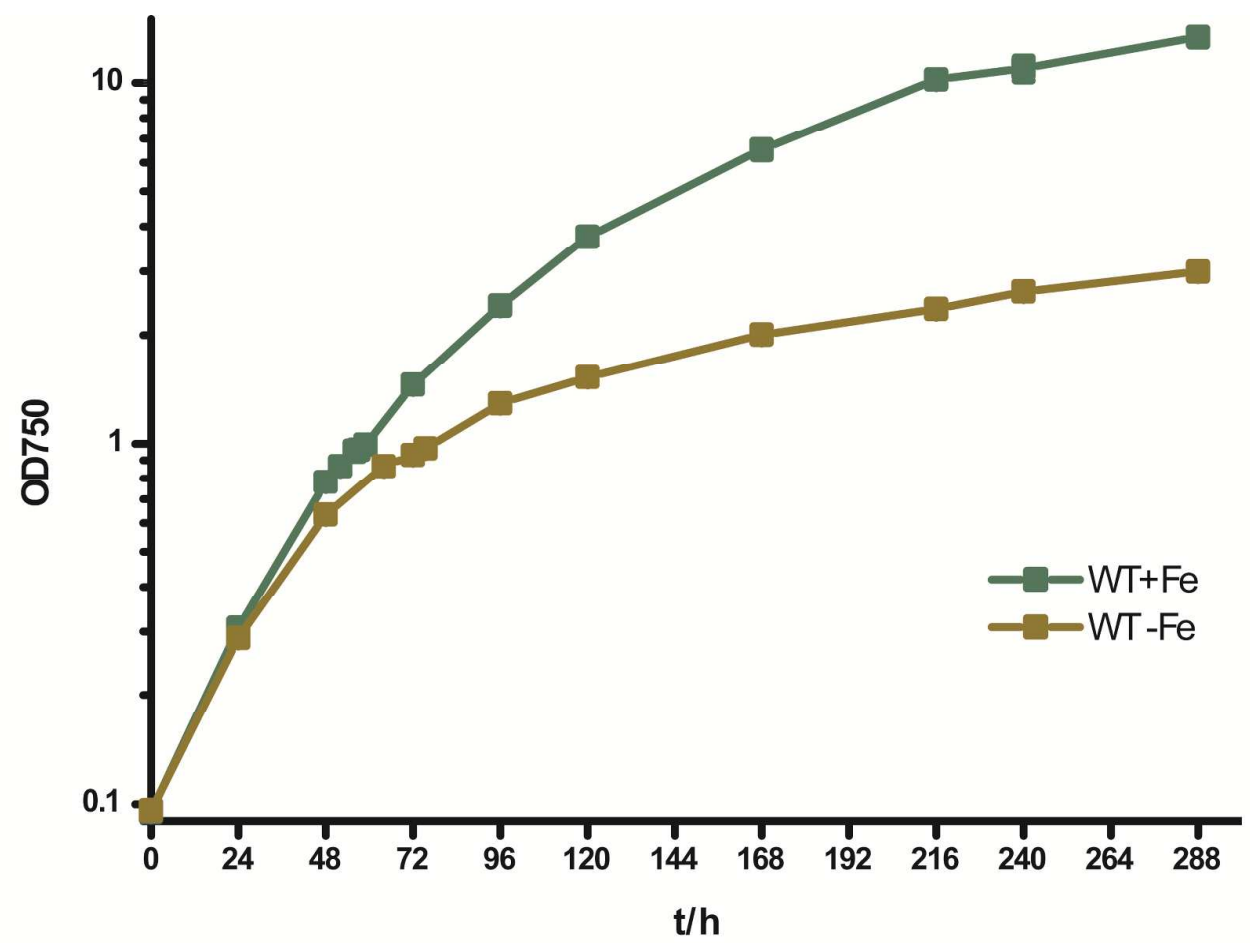

Figure S-1: Growth of Synechocystis sp. PCC 6803 under iron deprivation. The samples were collected at $\mathrm{OD}_{750 \mathrm{~nm}}=1(\sim 70 \mathrm{~h})$ in both iron sufficient $(\mathrm{WT}+\mathrm{Fe})$ and iron deprived (WT-Fe) conditions and after 12 days $(288 \mathrm{~h})$ under iron deprivation. The cultures were grown at $1 \% \mathrm{CO}_{2}$ atmosphere with white light illumination of 50 $\mu \mathrm{E}$. 


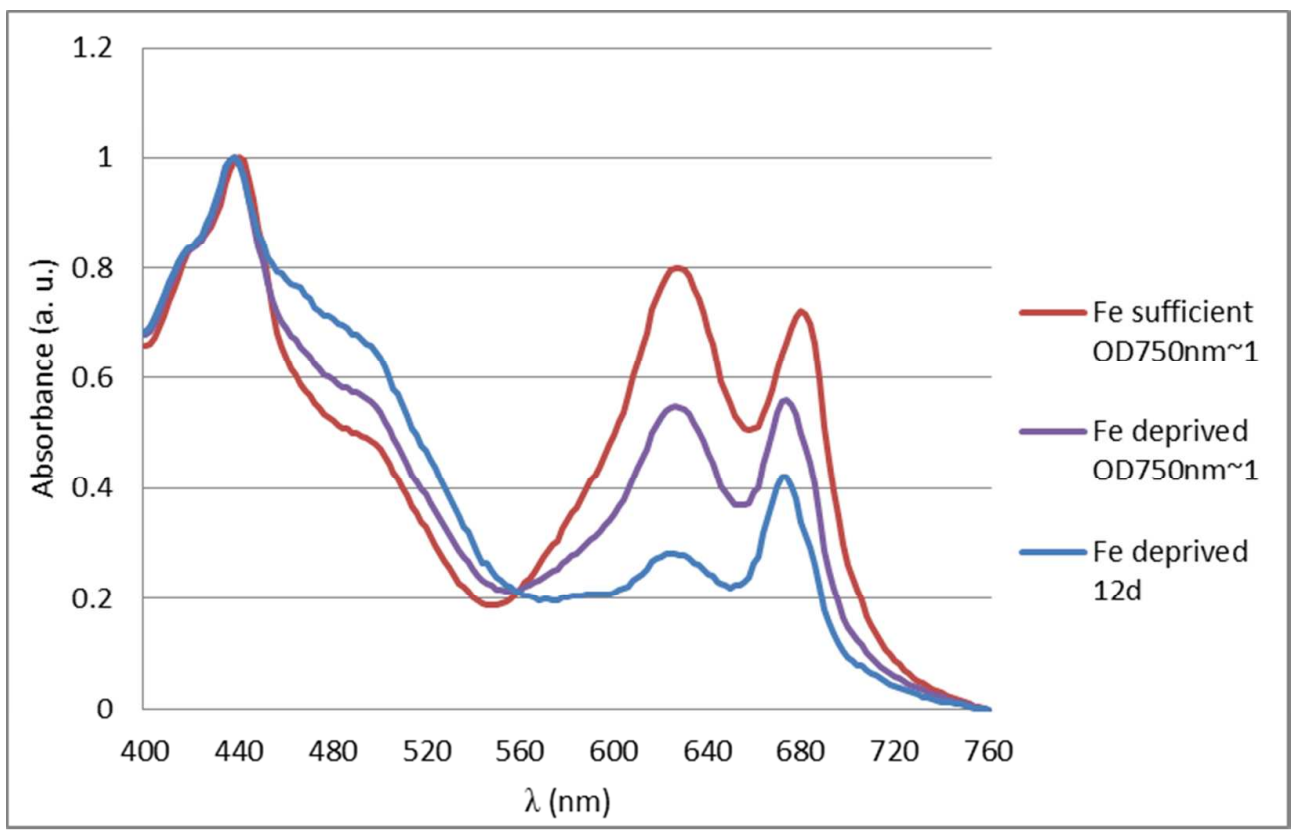

Figure S-2. In vivo absorbance spectrum at $\mathrm{OD}_{750 \mathrm{~nm}} \sim 1$ under standard (red line) and iron deprived (purple line) conditions as well as at $12 \mathrm{~d}$ time point (blue line) under iron deprivation. In both time points, the typical iron starvation induced chlorophyll a shift (due to the accumulation of IsiA) from 679 to $673 \mathrm{~nm}$ can be seen as well as lower phycobilin absorption peak at $625 \mathrm{~nm}$ and higher carotenoid absorption peak at $485 \mathrm{~nm}$. The red line represents the iron sufficient and the purple line the iron deficient cultures. The spectrum is normalized to maximum and minimum values.

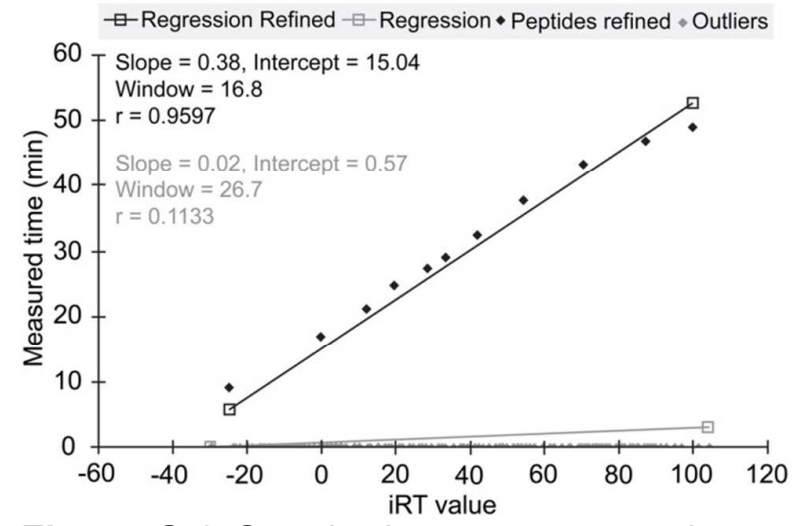

Figure S-3 Standard curve representing retention times of the iRT-peptides in the initial alignment run. 


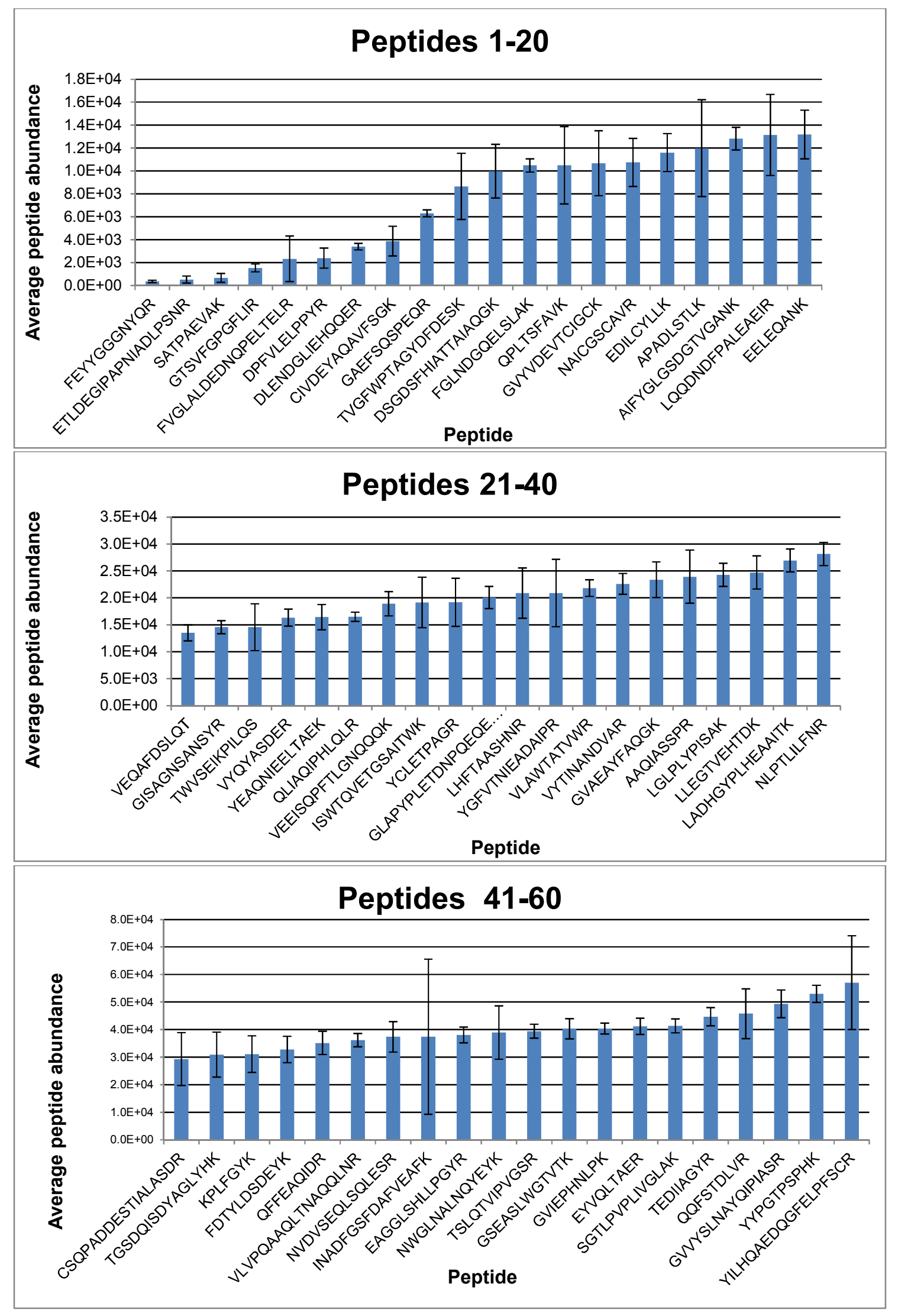




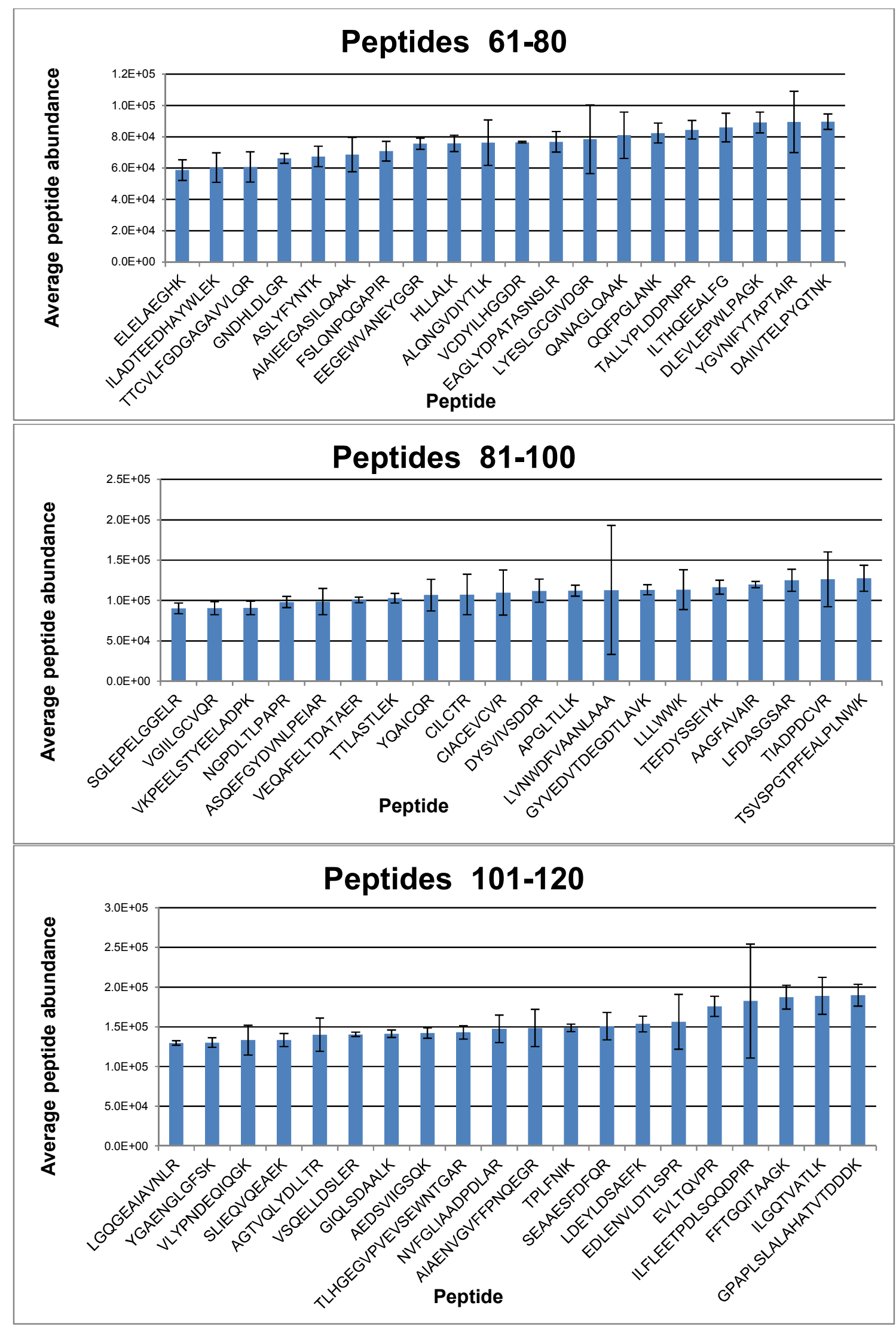




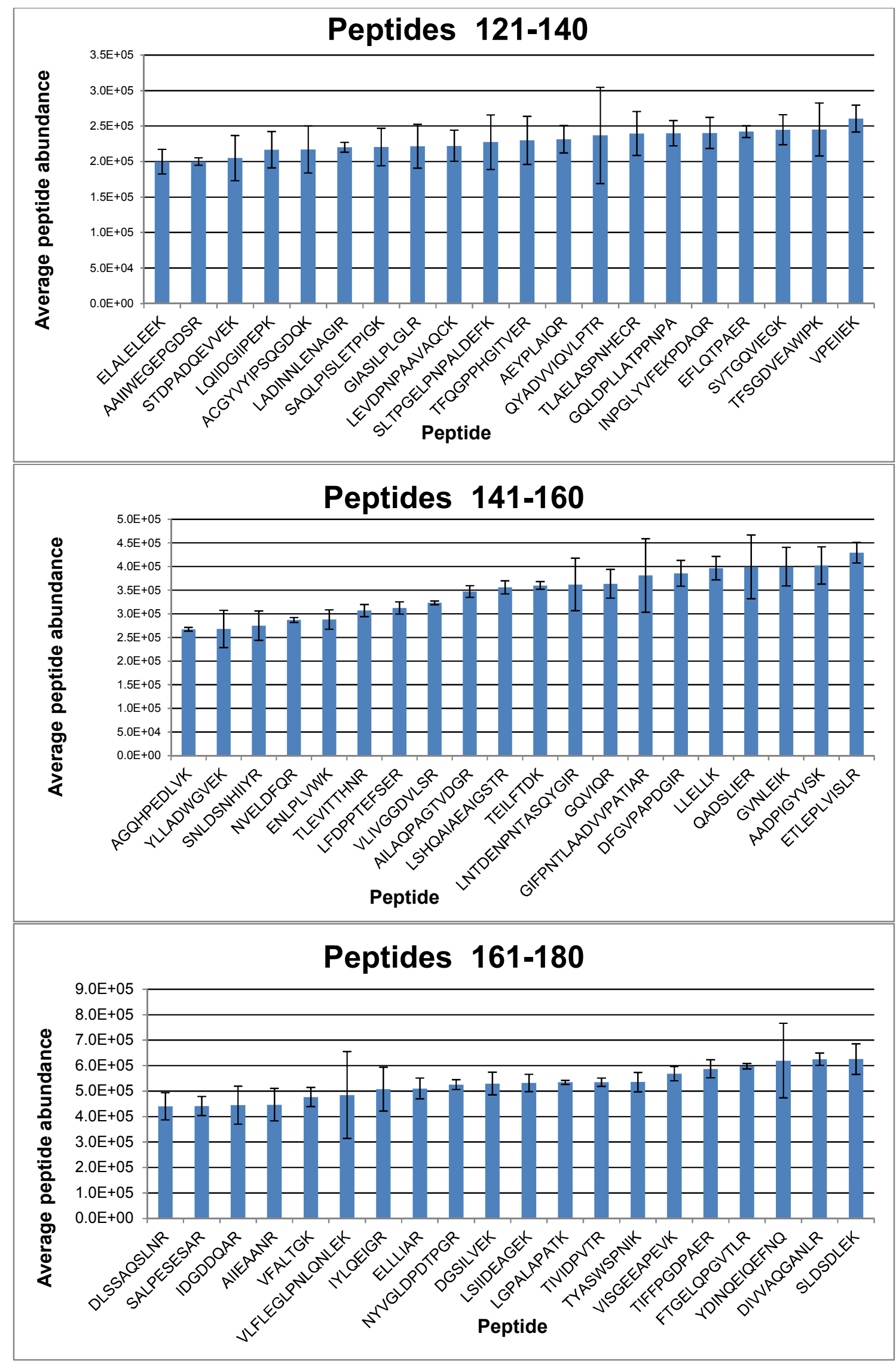




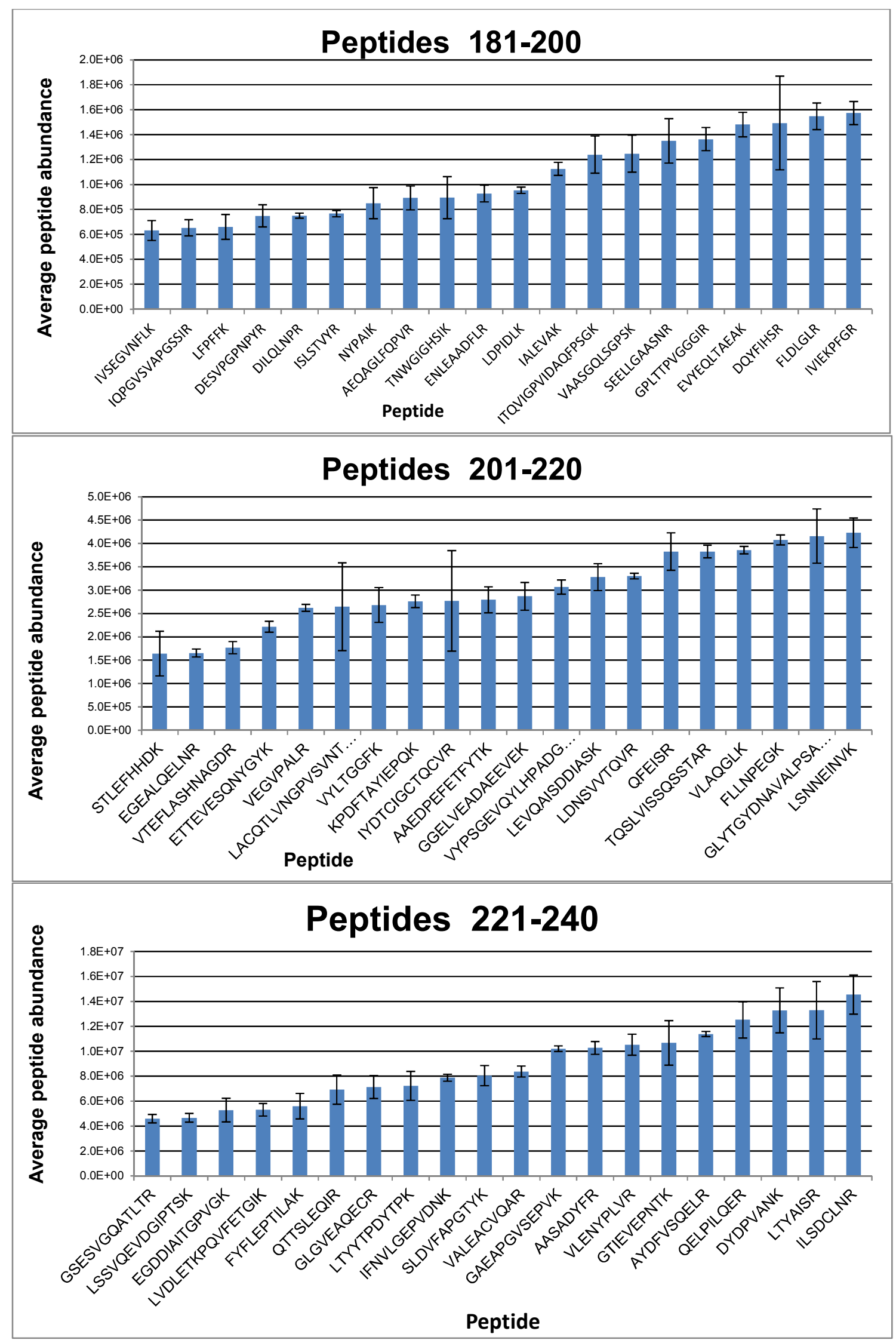




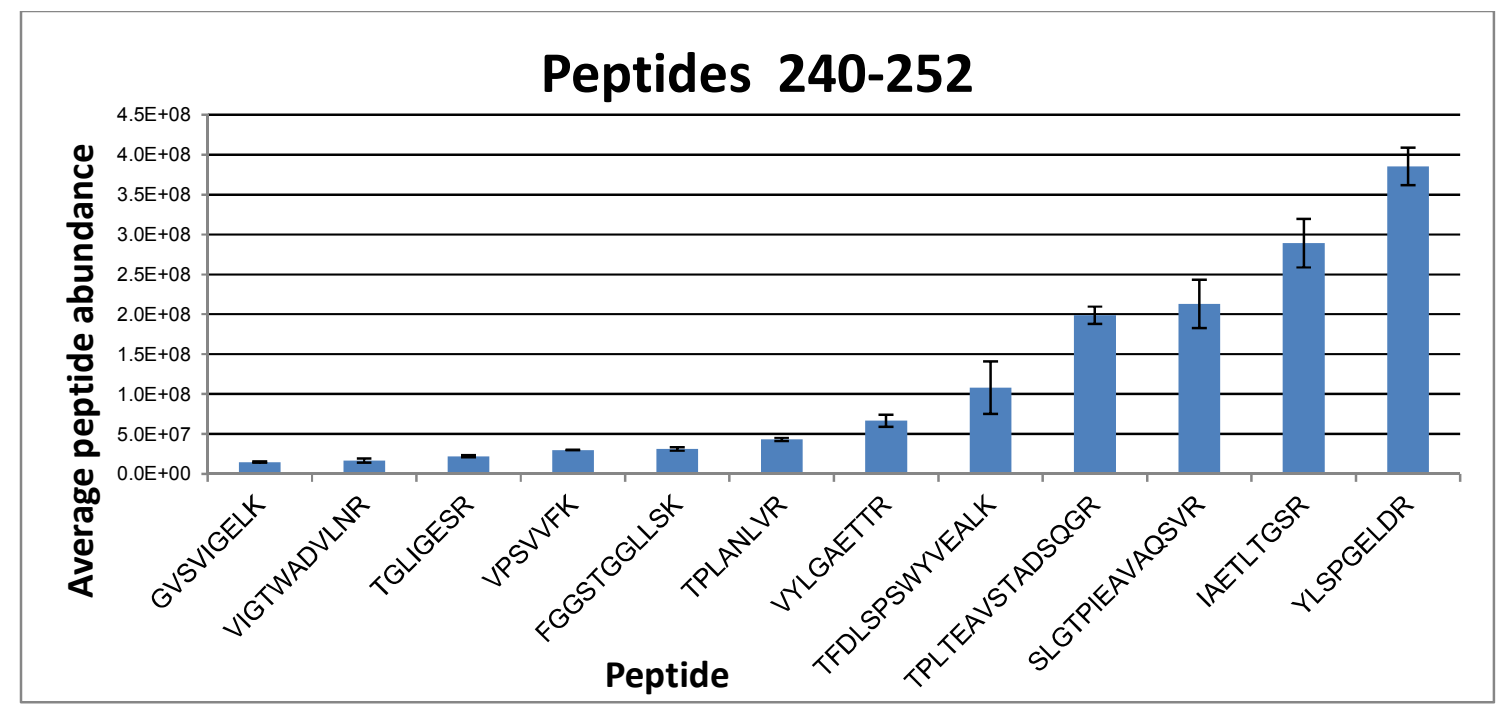

Figure S-4 Abundance of 252 peptides with CVs calculated for 3 biological replicates of wild type in standard condition.

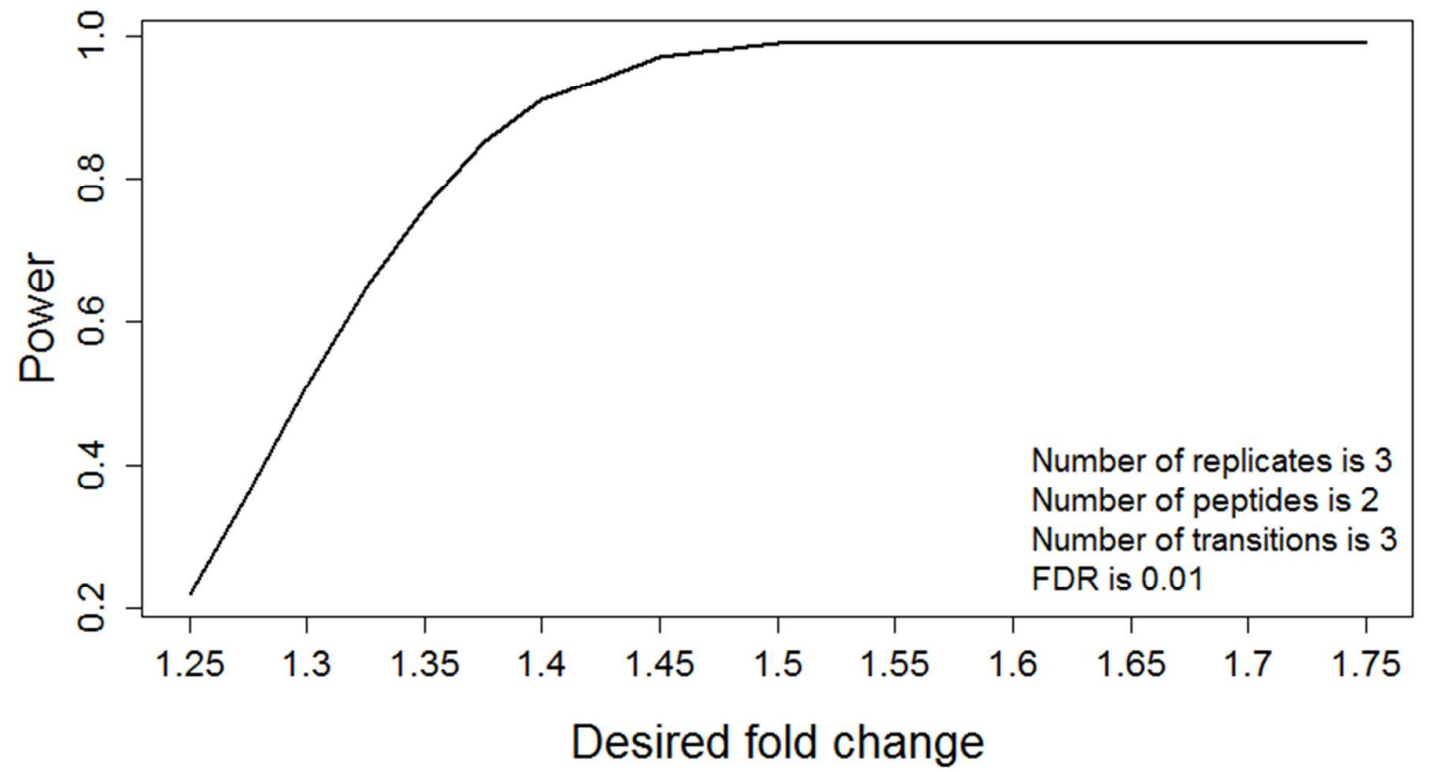

Figure S-5 Estimation of significance of the expression level change (FC) in the experiment. 